\title{
Archéopages
}

Archéopages

Archéologie et société

43 | 2016

Médecines

\section{Fouiller et lire les décors peints pour révéler l'architecture}

\section{Méthodologie appliquée à l'archéologie préventive}

Digging and reading painted decors to reveal architecture. Methodology applied to preventive archaeology

Excavar y leer los decorados pintados para revelar la arquitectura. Metodología aplicada a la arqueología preventiva

\section{Julien Boislève}

\section{OpenEdition \\ Journals}

\section{Édition électronique}

URL : https://journals.openedition.org/archeopages/2368

DOI : 10.4000/archeopages.2368

ISSN : 2269-9872

\section{Éditeur}

INRAP - Institut national de recherches archéologiques préventives

\section{Édition imprimée}

Date de publication : 1 novembre 2016

Pagination : 90-101

ISSN : 1622-8545

\section{Référence électronique}

Julien Boislève, «Fouiller et lire les décors peints pour révéler l'architecture », Archéopages [En ligne], 43 | 2016, mis en ligne le 01 décembre 2018, consulté le 04 juin 2021. URL : http://

journals.openedition.org/archeopages/2368 ; DOI : https://doi.org/10.4000/archeopages.2368 


\title{
Fouiller et lire les décors peints pour révéler l'architecture Méthodologie appliquée à l'archéologie préventive
}

\author{
Julien Boislève Inrap, UMR 8546 «AOROC»
}

\begin{abstract}
À la faveur du développement de l'archéologie préventive et d'une meilleure prise en compte progressive des peintures murales, les techniques de prélèvement et d'étude de ce mobilier ont été développées et adaptées à l'Inrap pour mieux répondre aux contraintes de ces fouilles, sur l'ensemble de la chaîne opératoire, du diagnostic à la publication. Sous réserve de l'application d'un protocole de fouille adéquat, l'analyse toichographologique apporte des informations variées sur les architectures et leurs évolutions.
\end{abstract}

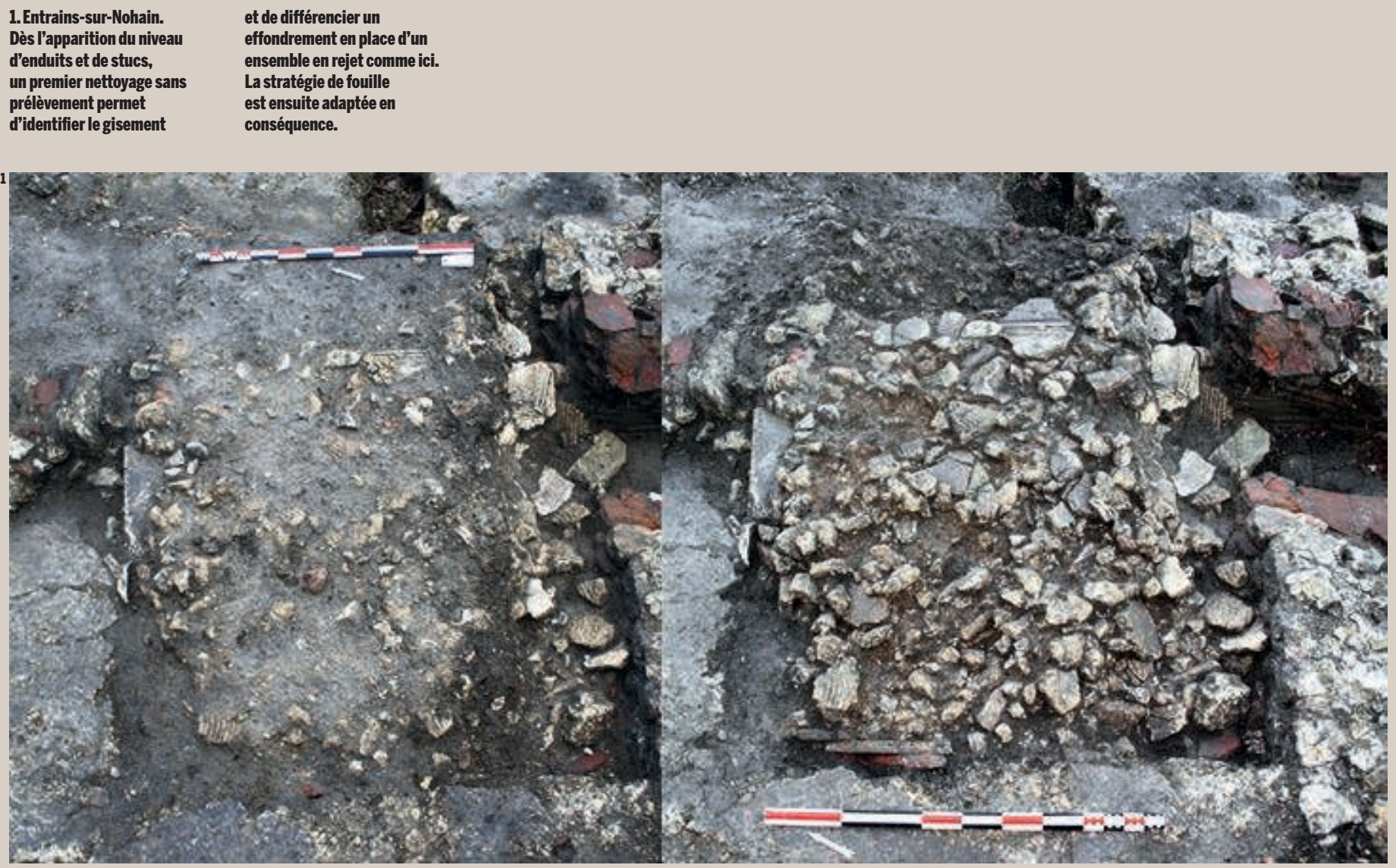


La progression du nombre d'études, publications et expositions touchant à la peinture romaine témoigne bien de l'importance que revêt aujourd'hui l'analyse toichographologique et de l'évolution des pratiques vis-à-vis de vestiges longtemps jugés ingrats et dont on estimait souvent, à tort, ne pas pouvoir tirer grand-chose. Parmi les différentes études de mobiliers décoratifs (mosaïques, placages et opera sectilia, enduits peints et stucs), la toichographologie, qui s'attache spécifiquement aux revêtements d'enduits stuqués et peints, a mis en place depuis plusieurs décennies des méthodologies de fouilles et d'étude. En France, la multiplication des prescriptions de fouilles intégrant la gestion des peintures a imposé de mettre ces méthodes à l'épreuve de l'archéologie préventive ${ }^{\mathbf{1}}$, de les adapter et de les perfectionner pour mieux répondre aux contraintes des fouilles ${ }^{2}$. Il faut d'emblée garder à l'esprit les interactions avec les autres formes de décor, celle des sols notamment, mais aussi les placages muraux avec lesquels les peintures cohabitent souvent. Ce constat implique donc une surveillance particulière de l'ensemble de ce mobilier en fouille et la réalisation d'études spécialisées qui gagnent à ne pas être totalement autonomes et à donner lieu à un recoupement systématique pour une confrontation des résultats.

\section{Le diagnostic, une évaluation déterminante}

La phase de diagnostic est déterminante, notamment pour une discipline qui nécessite souvent la présence d'un spécialiste dès la phase de terrain. Si les moyens n'ont pas été anticipés et que la présence d'enduits n'a pas été clairement mentionnée dans la prescription, il devient alors difficile d'obtenir les services d'un spécialiste lors de la fouille et encore davantage de dégager les temps d'étude nécessaires en postfouille ${ }^{\mathbf{3}}$.

Dans le cas de peintures, toute la difficulté consiste à identifier précisément le gisement et à évaluer l'étendue des surfaces à traiter sans mettre en péril la cohérence des vestiges pour une fouille ultérieure. Chaque cas nécessite d'adapter la stratégie, mais quelques grandes lignes directrices peuvent être retenues pour guider les opérations.

chantiers d'archéolog

préventive intégrant

des enduits sur toute

la chaîne opératoire

fut mené à Nîmes,

en 2006-2007, parking

Jean-Jaurès, sous

la direction de Jean-Yves

Breuil, Inrap.

2. Les questions

de restauration et de

muséographie seront

ici laissées de côté.

3. Le travail de

postfouille, du lavage

au rapport, est en

moyenne estimé

à un jour par caisse.

C'est donc davantage

le volume de caisses

à traiter, souvent

important, qui engendre

des temps d'étude

conséquents.

\section{Identifier la nature du gisement pour adapter la stratégie de fouille}

Qualifier précisément à quel type de vestige d'enduit on est confronté entraîne une stratégie différente selon les cas. Trois configurations de gisements peuvent être rencontrées : les enduits peuvent être retrouvés en place sur le mur (enduits in situ), ou en plaques cohérentes dans la pièce d'origine (« effondrés en place»), ou encore en position « tertiaire» (effondrés ou démontés puis évacués, réutilisés...), remaniés (fragments plus ou moins épars, coupés de leur contexte d'origine précis).

L'exercice d'identification reste toutefois difficile sur une surface parfois restreinte, alors que la fouille en aire ouverte permet justement de mieux percevoir le gisement [ill. 1]. Le dégagement mécanisé d'un niveau d'enduits nous prive assurément de la lecture de la nature du gisement, sans compter les dégâts irréversibles occasionnés sur le mobilier et sur la cohérence de sa disposition en lien avec le contexte architectural.

Dans le cas d'enduits remaniés, les fragments se présentent sans cohérence évidente, c'est-à-dire qu'ils sont en tous sens et ne forment pas des plaques en connexion. Plusieurs décors peuvent être mêlés, ce qui demeure parfois difficile à identifier dès la fouille. La densité peut être extrêmement variable, de quelques fragments épars dans une couche de remblai à un niveau de rejet exclusivement composé de milliers de fragments d'enduits. Sans l'expérience d'une lecture fine de la position des fragments entre eux, la confusion peut donc être aisée entre un niveau effondré en place et une densité d'enduits placés au rebut. Dans la première situation, le niveau est cohérent sans se présenter pour autant de manière simple. L'effondrement peut avoir eu lieu d'un seul tenant et livrer alors une grande surface d'enduit, souvent de revers, en connexion et généralement bien à plat sur le sol ou les premiers niveaux de comblement de la pièce. Mais il peut aussi avoir été progressif ou violent, par exemple par un incendie, entraînant alors un chaos de plaques enchevêtrées, se présentant aussi bien de face que de revers, se recouvrant et se répartissant sur plusieurs dizaines de centimètres de hauteur au pied des murs.

Le cas des enduits en place pose moins de difficultés d'identification. Le décapage progressif conduit souvent à voir apparaître une ligne continue de mortier qui signale un mur. On notera toutefois que, parfois, seul l'enduit est conservé en place alors que les matériaux du mur ont été récupérés. En surface, on veillera à ne pas écrêter le niveau d'apparition de l'enduit. Une passe mécanique trop basse et la seule disparition d'1 cm de hauteur de l'enduit en place annulera toute chance de reconnecter les parties supérieures si d'éventuels effondrements en place lui sont associés dans la pièce.

\section{Prélever, un choix délicat...}

Se pose ensuite la question de savoir jusqu'où pousser la fouille dans le cadre d'un diagnostic. Dans certaines situations, il est nécessaire de prélever les enduits pour accéder aux niveaux inférieurs et évaluer ainsi la puissance stratigraphique du site ; dans d'autres, il est préférable de préserver le gisement en l'état pour ne pas compromettre sa cohérence qui sera bien mieux abordée lors de la fouille.

Sur un niveau remanié, un sondage partiel avec prélèvement exhaustif des fragments est envisageable. Il s'agit alors de prélever les fragments au fur et à mesure de la fouille. Dans le cas d'un effondrement en place, qui nécessite, pour livrer toutes les informations attendues lors 
2. Nîmes. La ligne d'enduit

marquant le mur est dégagée

en surface pour voir à la fois

l'étendue de la pièce et

la longueur des parements

d'enduit conservés.
3. Arles. Pour limiter le temps

d'exposition de l'enduit,

on peut conserver, depuis son

sommet et jusqu'au niveau

de sol, une bande de terre

de 10 à $15 \mathrm{~cm}$ au devant

de celui-ci. Elle maintient et

protège l'enduit en place de

la lumière et des intempéries,

le temps de dégager le centre

de la pièce. Une fois le niveau

de sol atteint, ces bermes

sont fouillées pour dégager

le décor.
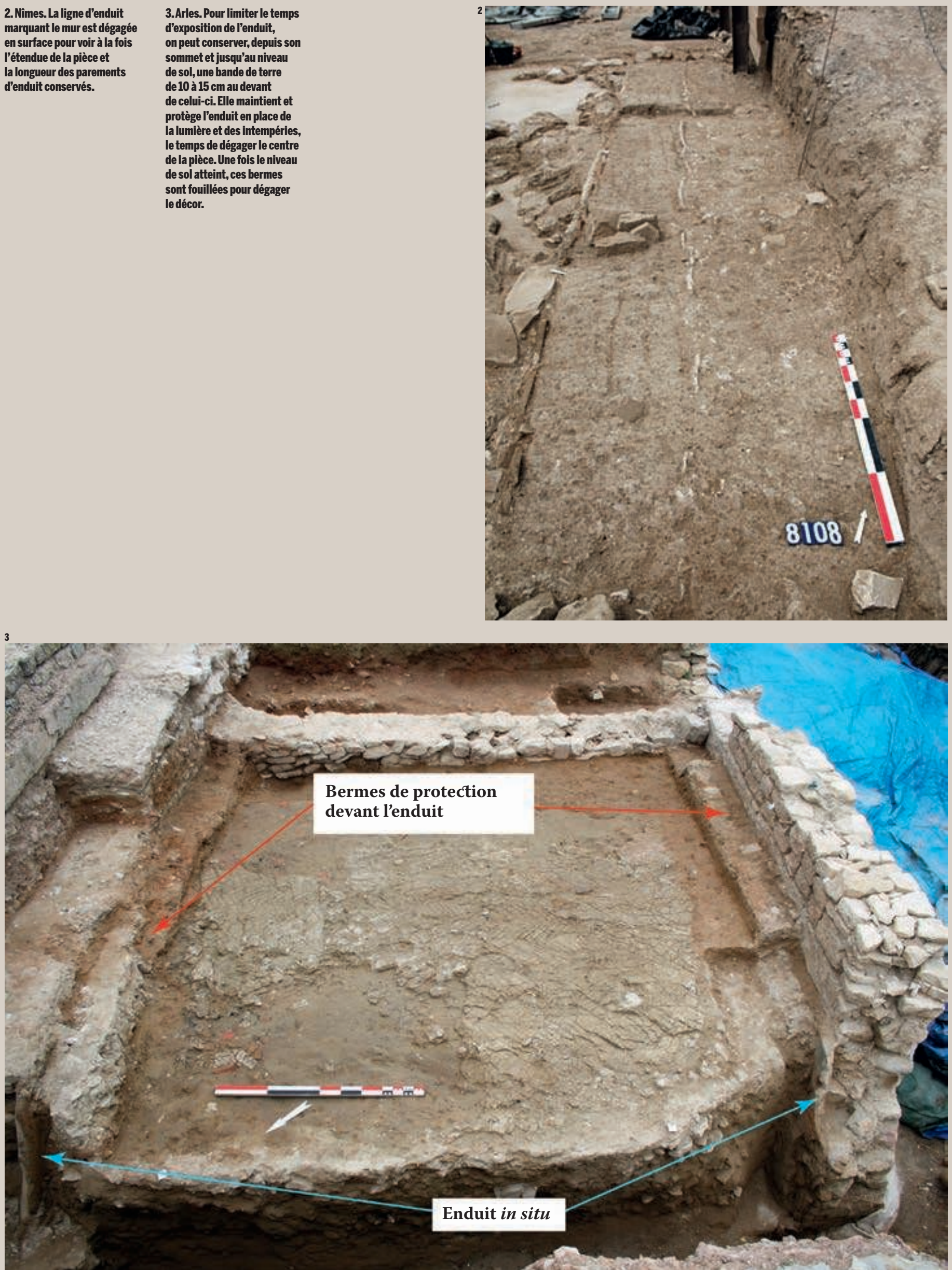
d'une fouille, d'être démonté en aire ouverte en abordant sa surface totale, un dégagement partiel suffisant à l'identification de la nature du gisement est préférable lors du diagnostic. La question du prélèvement est plus délicate. La dépose d'une partie de cet ensemble risque d'entraîner une perte d'information, chaque partie prélevée devenant difficile à raccorder par la suite aux déposes effectuées lors de la fouille, à moins, ce qui n'est que très rarement envisageable, de pouvoir disposer d'un spécialiste dès le diagnostic pour mettre en œuvre les méthodes adéquates ( $c f$. infra).

Mieux vaut donc envisager de préserver un effondrement en place dans son intégralité, la seule présence de tels vestiges étant la garantie d'un état de conservation déjà assez exceptionnel puisque la démolition du bâti est en partie conservée et que celui-ci devient potentiellement restituable en élévation. On peut essayer d'évaluer l'épaisseur du niveau à la faveur d'un endroit sans plaques. Dans le cas contraire, il est possible de décaler un sondage profond visant à connaître la stratigraphie à un autre endroit stratégique et qui n'impacte pas des couches d'enduits. Comme on ne percerait pas une mosaïque au diagnostic pour aller voir sa structure ou les niveaux sous-jacents, on ne perce pas un niveau d'enduits effondrés en place.

Concernant un enduit in situ, la prudence est également de mise. Dans le cadre du diagnostic, un dégagement test suffit à évaluer le potentiel des vestiges, leur état de conservation et leur hauteur tout en donnant une idée assez précise de la nature du décor. On conserve donc les remblais protecteurs en avant de l'enduit et on effectue un sondage avec dégagement sur une bande de $50 \mathrm{~cm}$ à $1 \mathrm{~m}$ de largeur, pour ne pas exposer la peinture et lui infliger deux phases de dégagement et un remblaiement.

\section{Documenter, renseigner, expertiser pour le rapport}

La documentation à prévoir au stade du diagnostic est nécessairement simple et rapide, tout en conservant les informations essentielles à l'évaluation du potentiel scientifique de l'ensemble. Un enregistrement du niveau d'apparition des enduits, de la surface aperçue et de sa position est utile. Des photographies nombreuses, d'ensemble et de détail, permettent souvent au spécialiste qui n'a pas toujours pu être présent d'établir les premières constatations et de rédiger la note d'expertise pour le rapport. On renseigne également l'état de conservation des enduits, d'une part pour évaluer leur potentiel scientifique, et d'autre part pour prévoir, le cas échéant, certaines opérations de consolidation ou certains protocoles de dépose qui peuvent être plus longs et plus coûteux et qu'il convient donc d'anticiper. On note la fragmentation de l'ensemble (en gros fragments ou très fissuré), la tenue des mortiers (solides à pulvérulents), l'état de surface (parfaitement lisible à fortement érodé).

Dans le rapport, une simple mention des enduits est insuffisante, tandis qu'une courte note d'expertise accompagnée de quelques photographies s'avère plus efficace. Il s'agit ici de donner au prescripteur les informations essentielles à l'évaluation du gisement pour déterminer l'intérêt scientifique et les moyens à mettre en œuvre. La nature du décor peut être exposée à partir de quelques indices clés. On essaye en revanche d'ouvrir sur les résultats potentiels que pourraient livrer la fouille puis l'étude de l'ensemble concerné et d'exposer les protocoles à respecter pour ce faire. Pour exemple, les notices d'expertise réalisées dans le cadre de deux rapports de diagnostic menés à Entrainssur-Nohain ${ }^{4}$ (Boislève, 2012 ; Boislève, 2013a) ont conduit à une prescription stipulant dans le cahier des charges que le responsable scientifique de la fouille serait secondé par un spécialiste des enduits peints.

\section{La fouille, prélever, observer et documenter Le décapage}

C'est la fouille fine des niveaux de démolition qui permet justement une bonne compréhension des architectures et une éventuelle restitution des élévations. Les enduits y sont généralement associés aux autres matériaux de construction. Dès l'apparition des niveaux d'enduits, mieux vaut passer à une fouille manuelle plus fine. Une passe mécanique trop profonde, ne serait-ce que de quelques centimètres, et c'est toute la cohérence d'un niveau d'enduit qui peut disparaître. Rappelons pour exemple qu'une paroi d'enduit tombée à plat sur un niveau de comblement ou un sol, ne représente qu'une couche d'1 à $5 \mathrm{~cm}$ qui peut très vite être irrémédiablement détruite par une intervention mécanique trop intrusive.

\section{Les enduits in situ}

Confronté à un enduit en place, on dégage le niveau d'arasement de l'enduit et du mur [ill. 2].

La récupération des matériaux du mur, à un moment où la pièce était déjà partiellement comblée, peut voir l'enduit rester en place malgré la destruction du support (récupération des pierres). Dès lors, il devient nécessaire de fouiller de manière inversée, c'est-à-dire de maintenir le comblement de la tranchée de récupération qui sert de nouveau support à l'enduit et de fouiller d'abord le comblement de la pièce.

Exceptionnellement, on fouillera donc les niveaux les plus anciens avant de plus récents.

En fonction de la nature du mur, l'enduit peut être particulièrement adhérent ou à l'inverse décollé du parement, quand les plaques ne sont pas déjà partiellement glissées, retenues seulement par les niveaux comblant la pièce. Un enduit in situ présente souvent un fort risque d'effondrement [ill. 3]. Fouille, nettoyage, documentation et éventuelle dépose sont donc à prévoir dans un délai de réalisation le plus court possible. Une fois le décor dégagé, on procède rapidement au

nettoyage puis à la documentation. Le premier
4. Diagnostics réalisés sous la direction de expertise des peintures par Julien Boislève. 


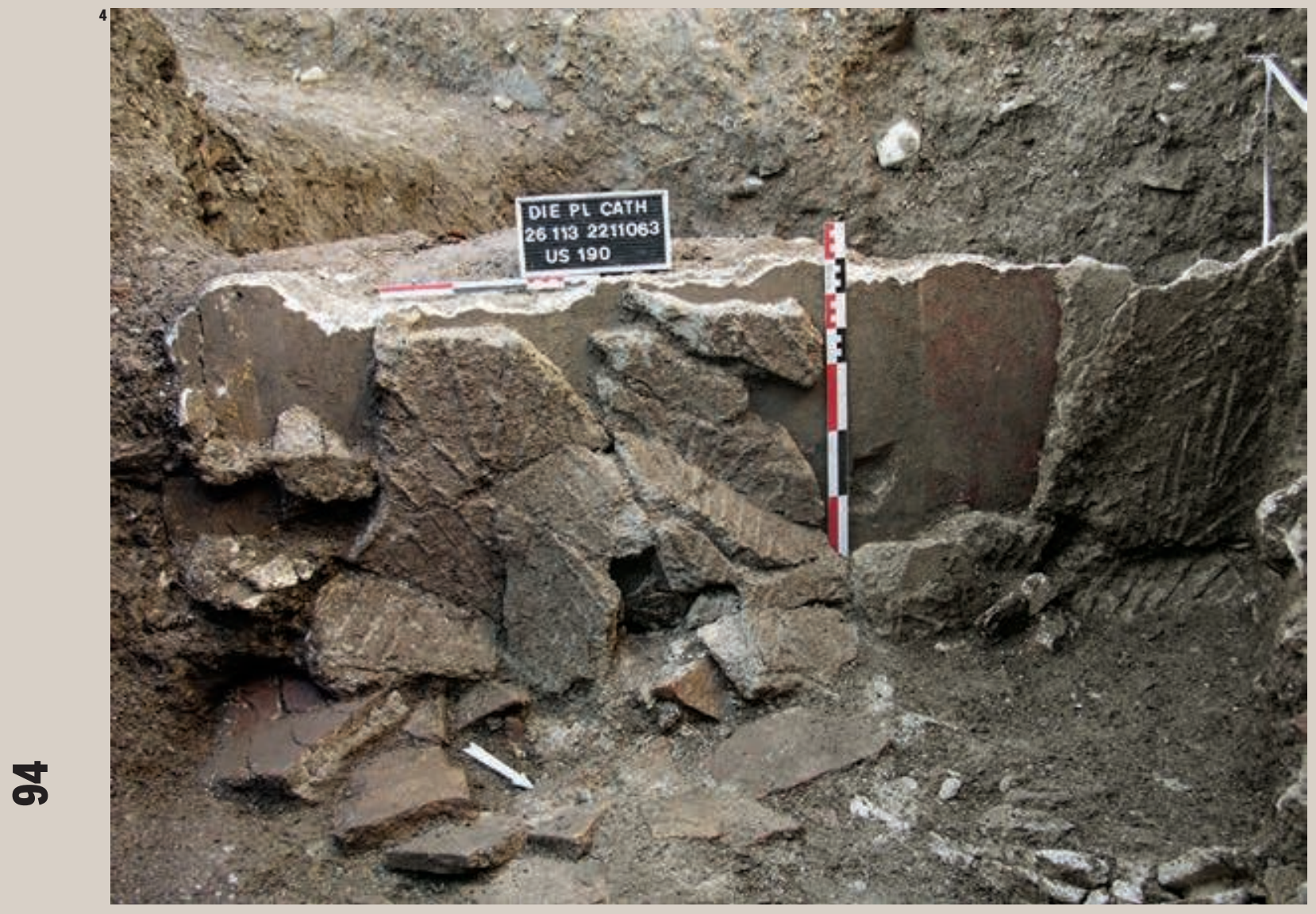

4. Die. Lorsque l'enduit in situ est associé à un effondrement en place, la dépose de la base du décor est indispensable pour l'étude ultérieure.

Les fragments pourront

éventuellement être

connectés à l'enduit déposé.

5. Nîmes. La zone

d'effondrement des peintures

est divisée en carrés d'environ

$1 \mathrm{~m}$ et les enduits prélevés

dans chacun. Les plaques

en connexion sont aussi

numérotées de manière

à avoir un véritable plan

de démontage.

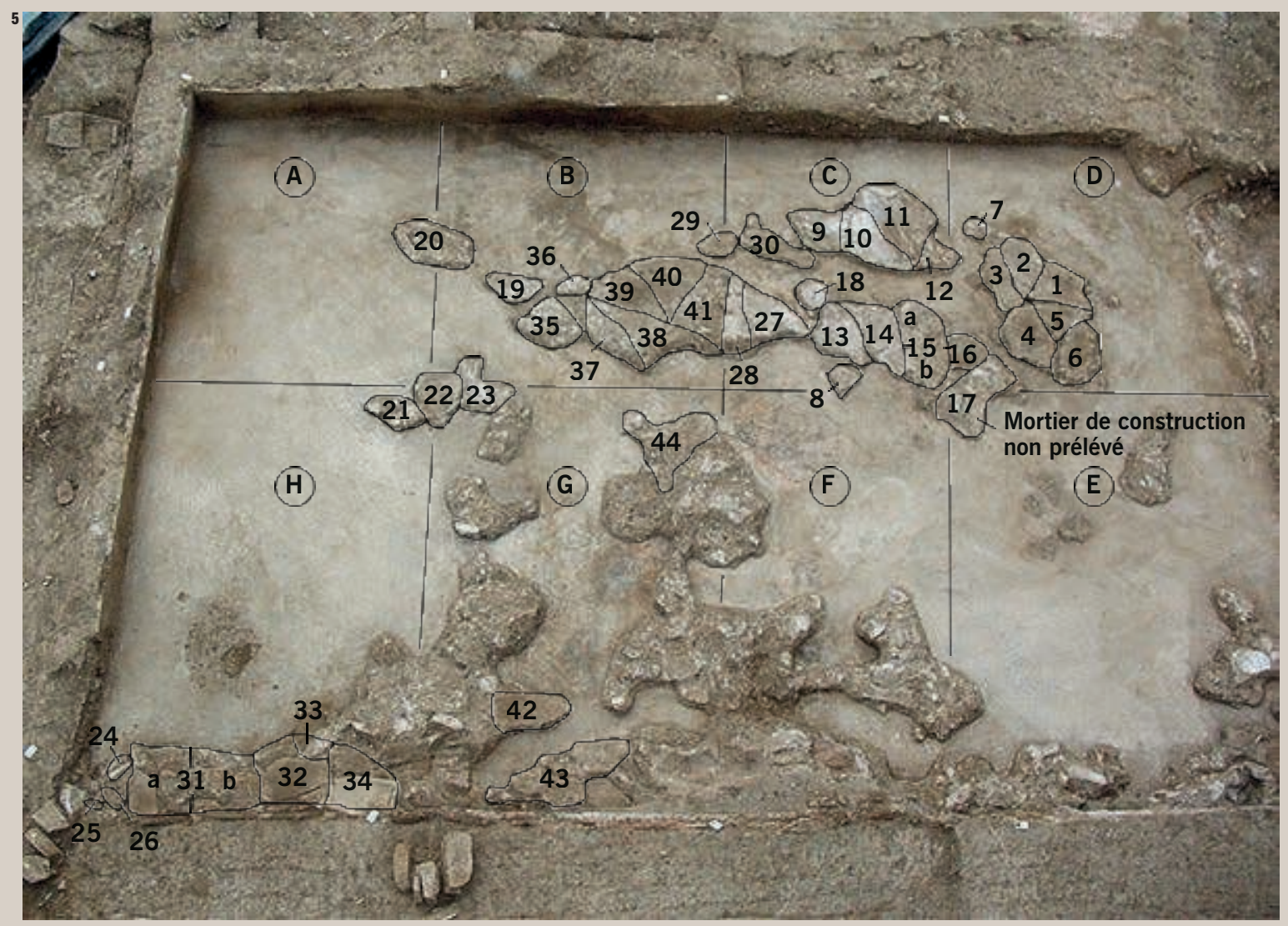


se fait d'abord mécaniquement. Délicatement, et en fonction de la bonne tenue du pigment et de la solidité du mortier, on élimine le plus gros de la terre résiduelle. Le nettoyage est ensuite affiné à l'eau. Il s'agit de rendre le décor parfaitement lisible en mettant bien en évidence les motifs. L'opération n'a toutefois pas pour but un nettoyage complet avec élimination des concrétions tel qu'il peut être effectué en laboratoire lorsque la peinture est destinée à être restaurée ( $c f$. infra).

Le relevé se fait d'abord par photographie avec humidification préalable au vaporisateur en prenant bien soin de multiplier les clichés de manière à disposer d'une vue d'ensemble montrant l'enduit dans son contexte, c'est-à-dire à l'échelle de toute la pièce. Une vue plus rapprochée couvrant l'ensemble du mur veille à disposer d'une couverture photographique parfaitement de face, indispensable en cas d'intégration à une restitution. Notons d'ailleurs que les techniques de prise de vue par orthophotograghie ou photogrammétrie peuvent s'avérer intéressantes.

Un relevé graphique est indispensable en complément du relevé photographique. Il s'effectue à l'échelle 1 sur un film plastique transparent tendu au-devant de l'enduit. Le relevé au feutre dessine le contour de l'enduit, les fissures principales puis le contour de chaque motif. On indique le niveau de sol et son altitude ainsi que la position précise de l'enduit par rapport à des éléments fixes de l'architecture pris en topographie (par exemple les angles de la pièce). Le relevé permet souvent de mettre en évidence certains motifs, incomplets ou effacés, que la photographie ne parvient pas à faire apparaître.

Un enregistrement traditionnel complète les données en décrivant l'état de conservation, le décor avec les mesures précises des éléments clés, les épaisseurs de mortier et toute information utile (pose de l'enduit avant ou après le sol, constitution des mortiers, raccords ou limites de travail...).

\section{La dépose}

Quel est ensuite le devenir de l'enduit? Tout vestige immobilier d'enduit peint n'a pas nécessairement vocation à être conservé. La conservation et donc la dépose d'un tel vestige (enduit, mosaïque ou autre) reviennent aux services de l'État. Trois cas justifient ou imposent une dépose. En premier lieu, lorsque l'enduit présente un intérêt du point de vue muséographique, on envisage la dépose si un musée local souhaite pouvoir présenter une peinture ou si le motif est suffisamment bien conservé pour être lisible aisément du public. Elle est également souhaitable lorsque la peinture in situ est associée à un effondrement en place [ill. 4]. Enfin, quand les conditions de terrain n'ont pas permis une bonne lecture du décor, un traitement en laboratoire, par exemple avec un nettoyage plus poussé, offre de meilleures chances de lire les motifs.
Selon les cas, l'acte de dépose peut nécessiter la présence d'un restaurateur. L'évolution rapide des méthodes et produits nécessite de bien définir le cahier des charges de cette opération et permet d'impacter de moins en moins la peinture. Le devenir et le traitement des déposes est anticipé en accord avec les différents acteurs (archéologues, restaurateurs, service de l'État et lieu de destination).

\section{Les peintures fragmentaires}

Le cas des enduits en position « tertiaire » est le plus aisé à aborder en fouille. Il autorise une fouille plus rapide. Malgré tout, certains ensembles, même en rejet - on peut citer ici le cas des stucs d'Autun (Boislève, Allag, 2011) s'avèrent particulièrement complets et permettent des recompositions conséquentes qui offriront de multiples données à condition de procéder à un prélèvement exhaustif. Les fragments sont ramassés au fur et à mesure d'une fouille suffisamment minutieuse pour ne pas endommager les enduits.

La gestion d'un effondrement en place, en revanche, nécessite la présence d'un toichographologue aguerri aux protocoles de prélèvements. Le dégagement des peintures effondrées impose un travail en aire ouverte, la fouille par moitié ou par quarts opposés nous privant d'une vision d'ensemble de l'effondrement et donc d'une compréhension de son organisation. Le prélèvement en connexion s'en trouverait également gêné. Si l'effondrement en place peut être assez simple, il est plus souvent chaotique, notamment lorsque plusieurs parois sont effondrées dans un même niveau. Le dégagement est manuel, souvent au petit outil, et vise, dans un premier temps sans prélèvement, à comprendre et à documenter l'organisation de l'effondrement. On ôte donc au maximum la gangue de terre scellant les enduits et on réalise ensuite une vue zénithale d'ensemble, si possible sur toute la pièce.

Une fois l'effondrement enregistré, le principe général du démontage consiste à conserver l'ensemble des connexions existantes sur le terrain afin de garantir des remontages conséquents et d'économiser un temps précieux lors de l'étude. Il s'agit en parallèle de localiser précisément les plaques par rapport aux murs, mais aussi les unes par rapport aux autres. Ces données sont indispensables à la compréhension précise de l'architecture puisqu'elles permettent de repositionner les plaques sur leur paroi. On procède à partir de la vue zénithale des enduits qui fait office de plan de démontage. La réalisation de films plastiques pour la dépose du fragmentaire nous paraît être beaucoup trop chronophage en contexte de fouille préventive. Elle n'est pas indispensable au prélèvement des plaques pour un spécialiste expérimenté.

Deux solutions simples permettent de localiser les peintures [ill. 5]. Il peut s'agir d'un carroyage, 


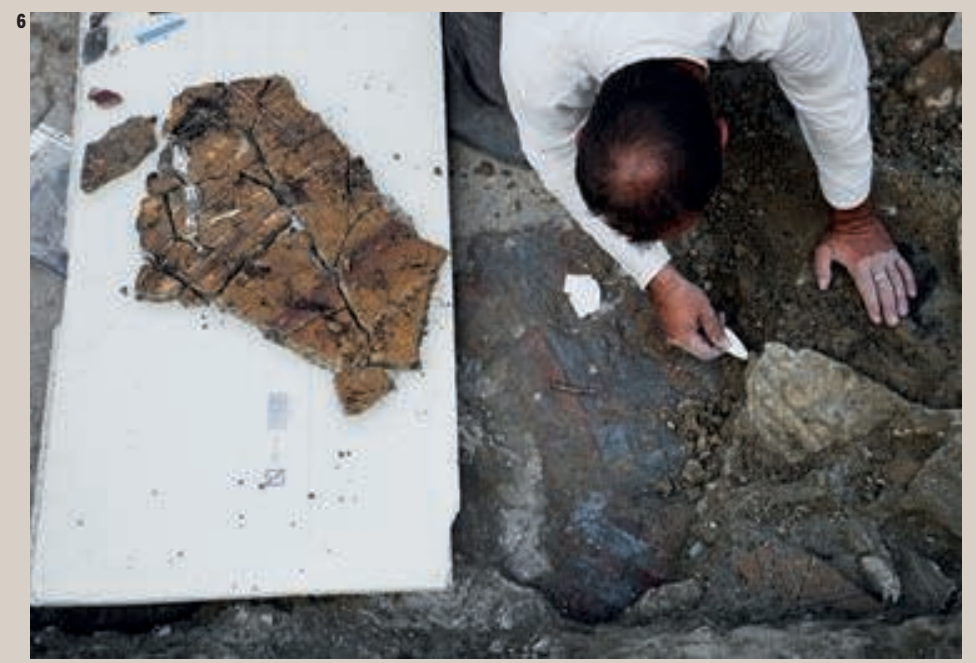

8

en prenant soin d'indiquer clairement la provenance de chaque caisse de prélèvement. Lors de l'étude, les connexions sont cherchées par cagette puis par carré et enfin entre les carrés voisins. On peut en revanche faire l'économie de recherches de connexions entre des fragments provenant de carrés trop éloignés, les peintures tombant au pied du mur et rarement à plusieurs mètres de distance. L'autre solution consiste en un plan de démontage où chaque plaque prélevée est numérotée sur la photographie de l'effondrement. En parallèle, un listing des caisses est tenu avec le numéro de chacune, la provenance des prélèvements et le numéro des plaques. Un inventaire est ainsi disponible à tout moment, permettant d'évaluer d'emblée le volume de mobilier à traiter en postfouille. Lors de l'étude, le plan de démontage permet de savoir facilement où se positionnait chaque plaque et avec quelles autres, de proximité immédiate, elle a des chances de pouvoir être remontée.

Le prélèvement à proprement parler dépend de la solidité des mortiers et de l'importance de la fragmentation. Si l'enduit offre une bonne tenue et que les plaques ne sont pas en trop petits morceaux, on privilégie un démontage manuel. Il consiste à déposer fragment par fragment en prenant soin de les repositionner en connexion dans la cagette de destination, opération bien plus complexe qu'il n'y paraît et qui demande une certaine expérience. Lorsque la plaque se présente de revers, on opère de même mais en retournant les fragments, ce qui complexifie la manœuvre [ill. 6].

Afin d'éviter les dégradations, on conditionne les fragments en connexion dans des cagettes de dimensions adaptées, stables et permettant une circulation de l'air (les moisissures entraînant des taches irréversibles). La manipulation restant

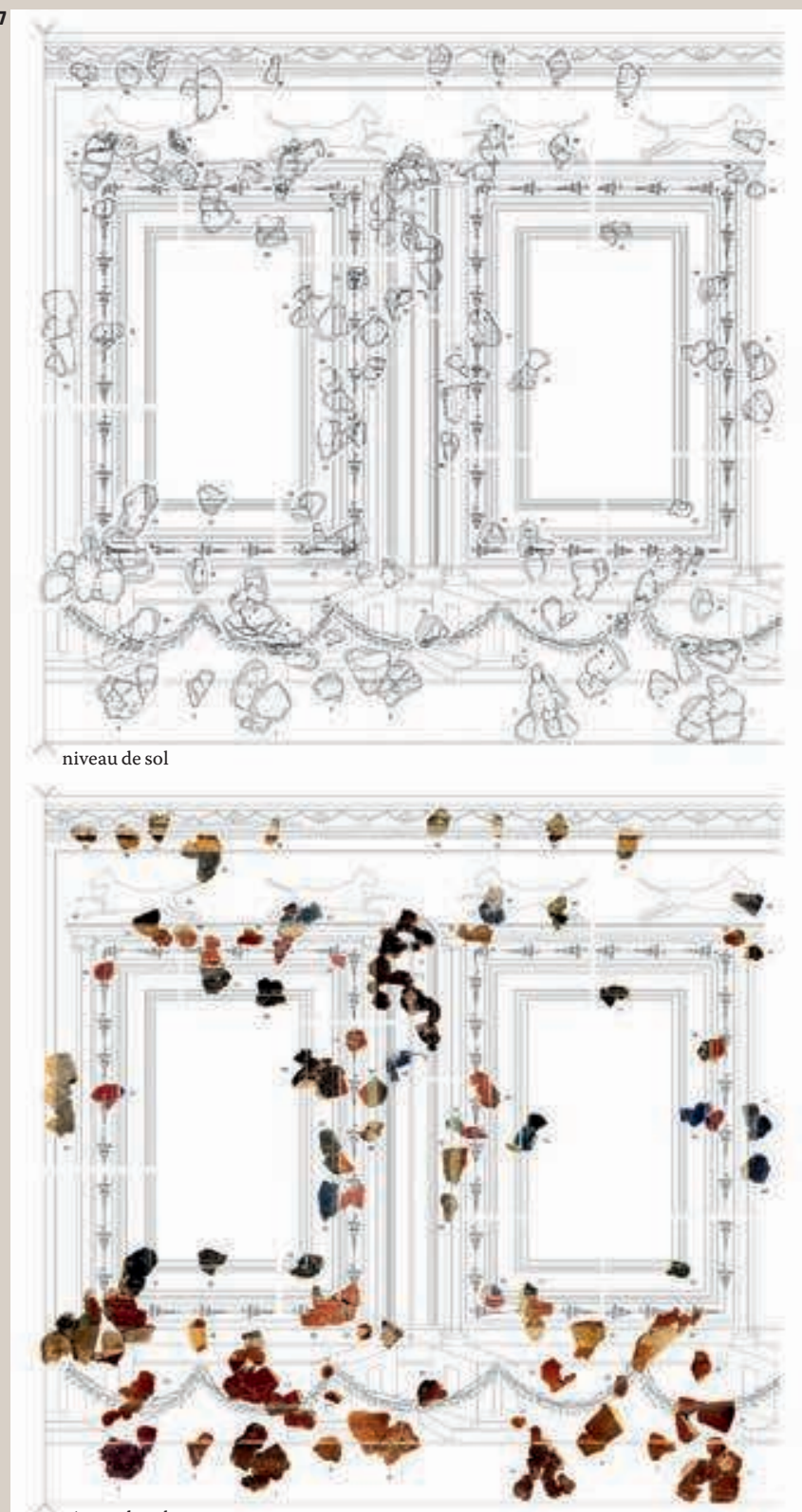

niveau de sol

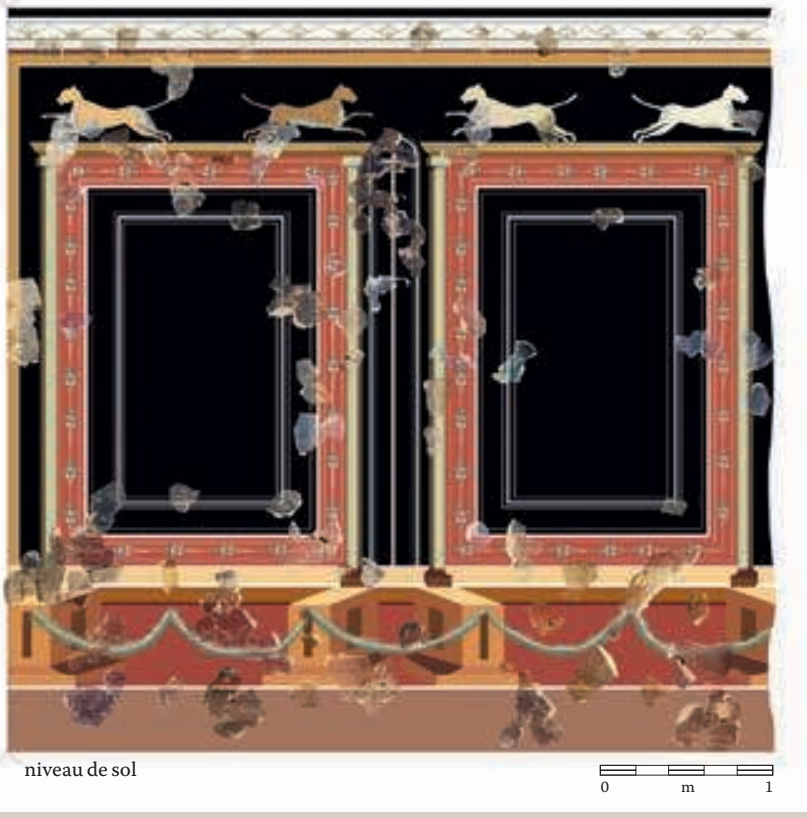




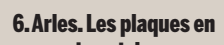

connexion et de revers sont retournées fragment par fragment et les fragments repositionnés au fur et à mesure en connexion sur une planche avant d'être conditionnés en cagette. 7. Bretteville-l'Orgueilleuse.

Les relevés scannés puis vectorisés, ainsi que les photographies des plaques, servent de fondement à l'élaboration éventuelle d'une restitution qui représente obligatoirement les éléments fragmentaires afin de pouvoir juger de la fiabilité d'une proposition.

5. Fouille menée par le musée départemental Arles antique, en partenariat avec l'Inrap, sous la direction de Marie-Pierre Rothé. 6. Fouille du parking Jean-Jaurès.

7. Site de Trémonteix fouille sous la direction de Kristell Chuniaud, Inrap. la cause principale de cassures et de perturbations des connexions, on réduit les risques de dommages en disposant les fragments soigneusement calés en une seule couche, face décorée visible, et en séparant bien les plaques en connexion les unes des autres.

Quand ils sont trop pulvérulents ou trop fragmentés, les enduits sont plutôt prélevés par encollage. Cette opération, réalisée par le spécialiste, est à envisager comme un dernier recours car la méthode nécessite un temps considérable de traitement en postfouille et contraint à apposer sur l'enduit des produits modernes dont nous maîtrisons mal le vieillissement.

\section{Des observations importantes dès la fouille}

La fouille est le moment où certaines observations clés doivent être menées. La disposition des effondrements et leur situation stratigraphique par rapport à certains autres matériaux de construction peut par exemple révéler la présence d'un étage comme nous l'avons identifié à Arles, Clermont-Ferrand ou Nîmes. Sur le site de la Verrerie à Arles $\mathbf{5}$ (Boislève 2014b), c'est la position altimétrique de chaque élément qui a prouvé l'existence de cette élévation. Au contact du sol de la pièce étaient effondrées des peintures de plafond puis des moulures de stucs courbes qui indiquaient un couvrement voûté. Les peintures murales du rez-de-chaussée étaient également partiellement effondrées. Au-dessus, un niveau de blocs de béton de tuileau à incrustation de tesselles marquait l'effondrement du sol de l'étage. Il était encore recouvert de très nombreux fragments de peintures dont des plaques en connexion qui provenaient de l'étage. À Nîmes ${ }^{\mathbf{6}}$ (Boislève et al., 2008 ; Boislève et al., 2011 ; Boislève, 2014a), l'effondrement d'un sol de béton était dans certains cas le signe le plus marquant de l'existence de l'étage, mais dans une autre domus ce sont les seuls décors qui constituaient la preuve de cette disposition architecturale. Sur le sol de la pièce, deux décors étaient effondrés en connexion. Leur sens de chute interdisait de supposer une provenance des pièces voisines, l'un des deux décors provenait donc forcément de l'étage. À Clermont-Ferrand ${ }^{7}$ (Boislève, Chuniaud, 2014), la nature des décors et leur effondrement en place constituaient une preuve. Dans une pièce à vocation artisanale, une grande plaque effondrée en connexion a livré un riche décor incompatible avec l'espace où il était effondré et dont les murs étaient recouverts d'un simple enduit de protection. La taille des plaques interdisait tout transport des peintures démontées et renvoyait donc là encore à l'étage du bâtiment.

La présence d'un spécialiste dès la phase de fouille permet également l'observation de certains indices ténus, importants dans la compréhension architecturale des lieux, mais qui ne peuvent être prélevés parce que trop fragiles comme des enduits sur terre. Ainsi, ces peintures bleues souvent signalées, mais qui n'ont pu être comprises comme des plafonds qu'à la faveur d'une observation stratigraphique et technique fine lors de la fouille de Nîmes, parking Jean-Jaurès. Des ensembles conséquents, à Arles par exemple, sont depuis venus confirmer cette observation.

\section{La postfouille, du lavage à la restitution}

Les fragments sont soigneusement nettoyés pour permettre une bonne lecture du motif et surtout des connexions nettes et franches. Le lavage à l'eau ne laisse pas tremper l'enduit dont le mortier est très absorbant. La brosse est utilisée sur les tranches et les revers, mais exclue sur la face peinte, lavée à l'éponge douce ou au doigt. Ce travail fastidieux constitue un moment d'observation privilégié de chaque fragment et est réalisé par le spécialiste ou tout au moins sous son contrôle.

Après séchage, la phase de remontage nécessite souvent de grandes surfaces de travail. Les plaques assemblées sont montées sur un lit de sable qui permet de stabiliser les fragments. Les fragments doivent être laissés libres car l'ajout de colle sur les tranches engendrerait des déformations importantes à l'échelle de grandes surfaces recomposées. Tout indice utile pour tenter d'assembler les milliers de fragments est exploité. On respecte tout d'abord la cohérence établie lors du prélèvement (remontages par caisse, puis par carré...) en suivant les plans de dépose. La forme des cassures et la cohérence des motifs sont les meilleurs indices, mais ils s'avèrent insuffisants pour réassembler les grands fonds unis pourtant indispensables pour disposer de mesures exactes et des enchaînements clés du décor. La lecture du sens de lissage, qui correspond à des fines stries laissées lors de la finition de surface, permet d'orienter les fragments et accroît considérablement les chances de connexions.

Une fois les remontages achevés, les plaques les plus importantes (même un simple fragment révélant un enchaînement inconnu ailleurs) sont sélectionnées puis mises à plat sur sable et photographiées. On évite les photographies non zénithales ou de fragments mal disposés et imparfaitement jointifs qui engendrent des distorsions et des déformations des motifs. Une multitude de photographies techniques ou de détail documentent en complément la facture de l'enduit ou les traces qui y sont perceptibles.

Le relevé graphique, à la différence de la photographie, n'engendre pas de déformations importantes. C'est donc sur ce document et sur une observation directe que l'on se fonde pour rétablir les dimensions du décor. Il est réalisé selon les mêmes procédés que pour les enduits in situ, c'est-à-dire sur film transparent. On note le contour des plaques, le motif, les tracés préparatoires, graffitis et toute information utile. Sur les photographies détourées comme sur 
8. Die. Le prélèvement

raisonné a permis de

repositionner précisément

chaque plaque et, sur la base

de $16 \mathrm{~m}^{2}$ de décor recomposé,

de proposer une restitution

offrant des mesures précises

et même l'emplacement

d'un meuble ayant laissé

une trace brûlée sur le mur.

\section{El Munts. Cet élément en}

cours de remontage

correspond à un plafond

à caissons dont les volumes

et décrochements complexes

n'auraient pu être envisagés

sans l'étude des peintures.

Au revers de celles-ci,

l'empreinte de la structure

de bois révèle le mode

de construction de ce type

de plafond.
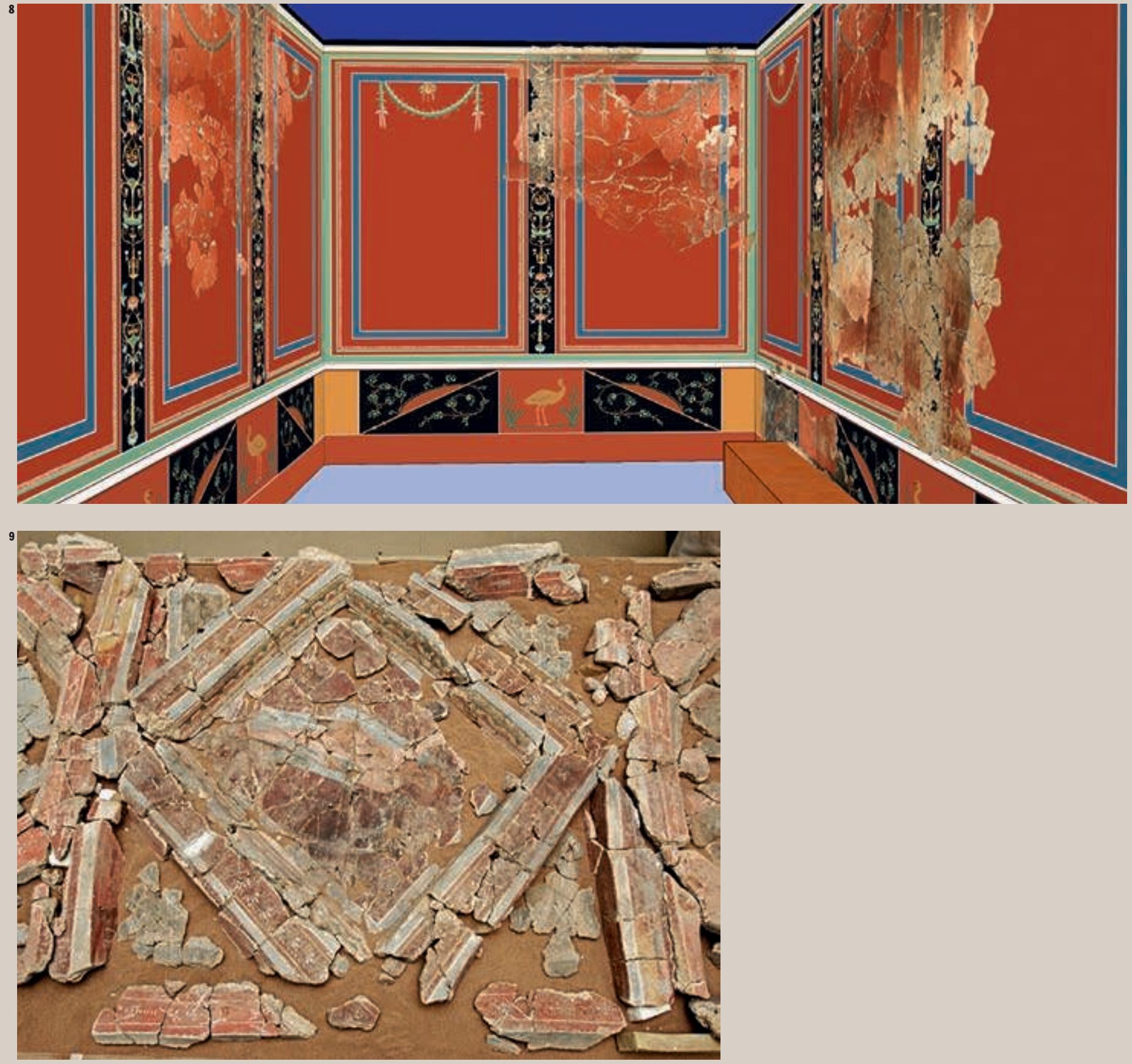
les relevés, on prend soin de conserver les zones de mortier et pas seulement le contour des zones peintes [ill. 7]. Les fragments ne sont souvent jointifs que par le seul mortier, dans l'épaisseur du fragment, alors que la surface peinte connaît des lacunes.

\section{L'architecture révélée}

Au-delà de la méthodologie, adaptable selon les cas et les moyens, il faut aussi exposer rapidement les résultats auxquels conduit l'analyse de ce mobilier. Nous en présentons ici les grandes lignes en nous concentrant sur l'apport architectural à partir de quelques études récentes. L'enduit est un moulage de l'architecture. Non récupérable à la différence d'autres matériaux de construction, il est abandonné sur place et devient notre meilleure chance de pouvoir connaitre les élévations disparues.

\section{La restitution des élévations}

À Die (Drôme) $)^{\mathbf{8}}$, la fouille d'une fosse de plantation d'arbre d'à peine $4 \mathrm{~m}^{2}$ a mis au jour l'angle d'une pièce où l'enduit était en place sur une hauteur assez importante, mais surtout les parties supérieures des parois effondrées en place ainsi que des plafonds sur terre (Boislève, 2014d ; Boislève, Ronco, 2016). Le prélèvement selon le protocole décrit a permis de comprendre le sens de chute et ainsi de restituer la pièce [ill. 8]. Sur un des murs, malgré une zone centrale très érodée où le décor n'était plus présent, tous les fragments ont été conservés puis réassemblés. Une grande plaque continue a indiqué la dimension de la pièce, soit 3,80 m sous plafond. Chaque fragment, même érodé, peut donc être précieux. La distance entre deux murs, dont un n'a pas été atteint par la fouille, a également pu être déterminée par les enduits et la lecture de l'effondrement. Sur le mur de refend, les panneaux de largeur différente et l'absence d'une alternance des bordures ajourées indiquaient la présence de seulement deux panneaux, confirmant donc la largeur de la pièce.

L'obtention de mesures aussi fiables repose bien sûr sur des remontages les plus complets possibles. On perçoit donc ici toute l'importance de cette phase d'étude où tous les fragments comptent et notamment les fonds unis. La recherche ne se focalise pas sur les seuls motifs.

La peinture murale peut ainsi mettre en évidence des pièces aux volumes imposants voire complexes. Issus d'un remblai, les stucs prélevés à Autun ${ }^{9}$ ont permis de proposer la restitution d'une pièce haute d'environ 5,75 $\mathrm{m}$ dont deux murs à angle droit sont connus (Boislève, Allag, 2011). L'un présente une série d'arcades peu profondes $(15 \mathrm{~cm})$ mais larges d'environ 1,30 m. L'autre offre des caissons et, en zone supérieure, des fenêtres à ébrasures. Ces dernières, très complètes, ont permis de mieux comprendre une vaste série de peintures qui présentaient un angle saillant et des arrachements en ressaut suivant une ligne de biais. Identifiées systématiquement à des cages d'escaliers à la suite d'une interprétation erronée sur des peintures de Chartres (Allag, Joly, 1995; interprétation révisée dans Allag, 2011, p. 572), elles sont en fait des éléments des tableaux latéraux d'une ébrasure de fenêtre. Le décor dévoile donc des volumes et met en évidence des jeux élaborés entre ouvertures réelles percées dans la structure du mur et ouvertures fictives dont le relief est créé de toute pièce par et pour l'ornementation.

Au-delà des pièces, l'enduit, et de manière générale le décor, peuvent nous renseigner sur l'architecture générale des lieux. Ainsi, à Clermont-Ferrand, dans le sanctuaire de Trémonteix, les enduits recueillis dans les deux fana donnent la structure générale de chacun (Boislève, Chuniaud, 2014). Les peintures effondrées en place dans un ambitus (passage étroit) entre les deux temples correspondent aux façades de ceux-ci avec un traitement très différent. Rattachés au temple nord, des enduits très érodés ne conservant presque plus leur décor constituent de longues plaques de profil hémicirculaire qui se raccordent aux éléments d'un pilier de section carrée, blanc et aux angles chanfreinés soulignés d'une bande verte. Ils révèlent une galerie de façade bordée d'une enfilade de piliers reliés par un mur parapet au sommet en chaperon arrondi. Le temple sud présentait un dispositif où les peintures montrent des colonnes maçonnées recouvertes d'un enduit traité à facettes et laissé blanc hormis une ligne de festons verts et jaunes [voir photo d'ouverture]. La galerie est donc bordée d'une colonnade ouverte, sans mur parapet.

De plans similaires, les deux temples auraient sans doute été restitués sur un modèle parfaitement équivalent si l'analyse des enduits n'était venue mettre en évidence toute leur différence. D’autres indices plus ténus, comme des enduits de façade conservant l'empreinte d'une toiture d'imbrices et de tegulae, indiquent le couvrement de la galerie par une toiture en appentis et le développement de la cella sur une hauteur supérieure.

\section{Les plafonds, voûtes et autres couvrements}

Le couvrement des pièces est également mis en évidence par l'analyse des enduits, avec des formes parfois variées au-delà de la simple voûte en berceau ou du plafond plat. À Rouen (Barbet, Lefèvre, 2007) ou à Reims ${ }^{\mathbf{1 0}}$, les enduits montrent une voûte d'arrête. À Mané-Véchen (Boislève et al., 2007) ou à Val-de-Reuil ${ }^{\mathbf{1 1}}$, une voûte surbaissée bordée de plates-bandes apparaît en couverture de couloirs. Et la disposition peut être encore plus originale ou complexe comme la voûte conique du laconicum de l'Île Sainte-Marguerite (Barbet et al., 1999) sur armature métallique ou le plafond à caissons et plates-bandes identifié dans la villa d'El Munts ${ }^{\mathbf{1 2}}$ (Guiral, Pelegrin, 2010, fig. 8) près de Tarragone [ill. 9].

fouille sous la direction

Inrap.

10. 72 Rue Ponsardin -

Yoann Rabasté, Inrap

Julien Boislève.
} 


\section{Les réfections et modifications}

Les réfections qui touchent la structure d'un mur affectent en général le décor. Si celui-ci n'est pas démonté à cette occasion, les travaux sont lisibles dans l'enduit conservé. Sur la fouille d'une domus du quartier Clérisseau à Nîmes ${ }^{\mathbf{1 3}}$ (Boislève, Cayn, 2013), une paroi tombée d'un seul tenant (une plaque de $5 \mathrm{~m}$ de longueur sur $3 \mathrm{~m}$ de hauteur) a permis de restituer le décor de la pièce et sa hauteur sous plafond de 4,80 $\mathrm{m}$. Grâce à l'étude de l'enduit ont pu être replacées des ouvertures avec une porte étroite percée à l'extrémité d'une paroi de division interne et qui desservait deux pièces à l'arrière. Deux larges baies rectangulaires étaient obstruées dans un second état de construction. Les surfaces des fenêtres rebouchées étaient enduites (on observe clairement la différence de mortier) puis peintes pour se raccorder au décor initial. Cette modification des ouvertures correspond sans doute à l'aménagement des pièces en arrière.

Souvent, les travaux ne correspondent toutefois qu'à une remise en état du décor à la suite d'une dégradation, comme au Thillay (Allonsius et al., 2013) où la voûte initiale, bleue à semis d'étoiles, est masquée par des concrétions puis couverte d'un second enduit. La réfection peut aussi marquer une évolution des modes ou des goûts des commanditaires. Souvent, le premier enduit est conservé et simplement piqueté pour l'accrochage du second décor. Â Nîmes, une des maisons livrait jusqu’à trois décors successifs.

\section{L'endroit et l'envers}

L'enduit est aussi riche d'informations architecturales par la lecture du revers, source précieuse pour caractériser la nature de la construction (Boislève, 2014c).

Dans bien des cas, le mortier garde une empreinte plus ou moins lisible de son support. Les exemples représentent à peu près tous les types de construction connus. Le mur de moellons laissera ainsi apparaître des rangs de carrés plus ou moins lisses et généralement disposés en quinconce [ill. 10]. On pourra même déterminer la nature des joints, par exemple tirés au fer comme à Bretteville-l'Orgueilleuse ${ }^{\mathbf{1 4}}$ (Boislève, 2013b). Les tubuli sont identifiés par l'empreinte de la largeur des conduits et du joint qui les sépare, mais aussi par les stries en croisillons caractéristiques de leur surface de pose. L'architecture en terre, si fréquente en Gaule, peut également être prouvée par la présence de stries d'accrochage en chevrons ou peignées et disposées en sinusoïdes, plus rarement par un piquetage qui laisse de petites excroissances arrondies au revers. Toutefois, la nature de la mise en œuvre, bauge, terre banchée ou torchis, est en général impossible à préciser sauf lorsqu'une forte chauffe a conservé une partie de la terre (Boislève, 2016). Enfin, l'enduit conserve aussi la trace de matériaux périssables comme les montants d'un mur à pan de bois, les roseaux ou lattes d'un dispositif de plafond ou les baguettes d'un clayonnage.

Cet aperçu rapide des apports primordiaux de l'analyse toichographologique s'est concentré sur l'architecture. Dans ce domaine, l'enduit est un atout majeur sinon, dans bien des cas, le seul indice tangible pour rétablir sur des bases fiables l'élévation d'un bâtiment. Néanmoins, il ne faut pas ignorer bien d'autres aspects non développés ici et qui livrent également des informations non négligeables pour caractériser les sites. L'analyse stylistique d'un décor permet de préciser une datation, d'évaluer le statut social d'un commanditaire, de hiérarchiser les espaces d'un habitat et de préciser certains aspects de la fonction d'une pièce. Les analyses techniques, sur les mortiers et les pigments, aident à mieux comprendre les gestes de cet artisanat. Les graffitis, fréquents sur ces surfaces, sont une source variée d'épigraphie ou de représentation témoignant de la vie quotidienne des lieux... autant de domaines et d'apports qui font des enduits peints un mobilier archéologique incontournable. Mais, nous l'aurons bien compris, ces données ne sont accessibles quà la condition de respecter l'ensemble d'une chaîne opératoire où des protocoles adaptés sont mis en œuvre. Le décor étant intimement lié à une architecture, il apparait indispensable, pour obtenir les meilleurs résultats, que le spécialiste soit présent dès la fouille pour percevoir la totalité des indices qu'il sait lui être utiles pour son analyse. Voir le lien entre l'enduit et le sol, comprendre la nature des murs, comment ils sont construits, saisir la disposition des bâtiments constituent autant d'observations qui font la différence dans notre capacité à rétablir les élévations de manière fiable. Si nous avons souligné une nette évolution des pratiques, les toichographologues travaillent encore trop souvent « en aveugle » sur des peintures totalement coupées de leur contexte. Les exemples cités, parmi d'autres, sont le fruit d'une collaboration étroite entre les divers acteurs d'un chantier de fouille.
13. Fouille sous la direction de Philippe Cayn, Inrap.

14. Le Bas des Prés, fouille sous la direction de Chris-Cécile BesnardVauterin, Inrap ; études des peintures, Julien Boislève. 


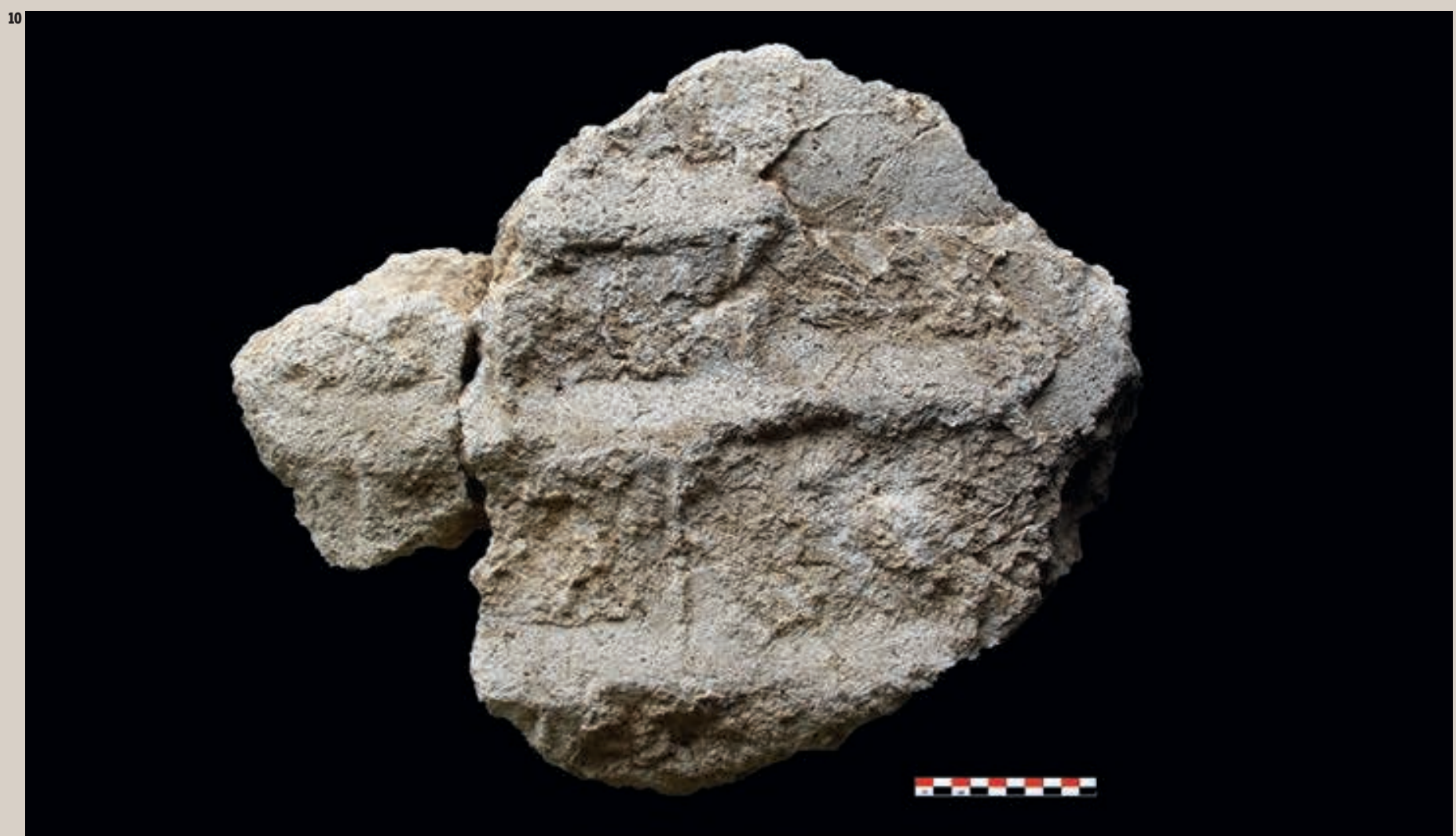

\section{Références bibliographiques}

Allag C., 2011, « Ouvertures, embrasures », in BALMELLE et al., 2011, p. 567-577.

Allag C., Joly D., 1995, « Les peintures murales romaines de Chartres (Eure-et-Loir), étude de quelques ensembles homogènes ", in Actes des séminaires de l'Association française de peintures murales antiques: Aix-en-Provence, Narbonne et Chartres, 1990, 1991, 1993, Revue archéologique de Picardie, $\mathrm{n}^{\circ}$ spécial 10, p. 169-187.

Allonsius C., Vermeersch D., Vibert-Guigue C., 2013, " La peinture de "voûte bleue étoilée" du bâtiment thermal du Thillay, au lieu-dit La VieilleBaune (Val-d'Oise) », in BoISLÈve et al., 2013, p. 327-341.

Balmelle C., Eristov H., Monier F. (éd.), 2011, Décor et architecture en Gaule entre l'Antiquité et le haut Moyen Âge. Mosä̈que, peinture, stuc, Actes du colloque international, université Toulouse IILe Mirail, 9-12 octobre 2008, Bordeaux, fédération Aquitania (coll. Aquitania Suppl. 20), 795 p.

Barbet A., Delamare F., Monier F., Vindry G. WALLET M., 1999, « Les peintures romaines de Léro au musée de la mer dans l'̂̂le Sainte-Marguerite (Îles de Lérins) », La Revue du Louvre et des Musées de France, 3, p. 37-46.

Barbet A., LefÈvre J.-F., 2007, « Peinture d'une piscine froide de Rouen, Station Métrobus, Palais de Justice », in Guiral Pelegrin, 2007, p. 429-432 et pl. 40

BoISLÈVE J., 2012, « Les enduits peints », in VINCENT G. (dir.), Bourgogne, Nièvre, Entrains-sur-Nohain, rue Romaine, construction d'un pavillon individuel Rapport d'opération, Inrap-SRA Bourgogne, p. 54-59.

BoISLÈVE J., 2013a, " Analyse des enduits peints », in Vincent G. (dir.), Bourgogne, Nièvre, Entrainssur-Nohain, 14, route d'Étais, construction d'un pavillon individuel, Rapport d'opération, Inrap-SRA Bourgogne, p. 74-78.

BOISLÈVE J., 2013b, « Les enduits peints », in BESNARDVAUTERIN C.-C. (dir.), Bretteville-l'Orgueilleuse, Basse-Normandie, Calvados, Le Bas des Prés, "Lotissement Résidence les Parcs », De la ferme à la villa, 1000 ans d'occupation : évolution d'un domaine agricole de la fin du premier âge du Fer à la fin de l'Antiquité, Rapport d'opération, Inrap-SRA BasseNormandie, vol. 2, p. 303-373, vol. 3, p. 186-331.
BOISLÈVE J., 2014a, « Nouvelle découvertes à Nîmes (Gard, France), 54 décors issus des fouilles du parking Jean-Jaurès et de la Percée Clérisseau ", in Zimmermann N. (éd.), Antike Malerei zwischen Lokalstil und Zeitstil, Actes du XI ${ }^{e}$ colloque de l'AIPMA, 13-17 septembre 2010, Éphèse, Vienne, p. 657-662, pl. CLXXXVII-CLXXXVIII.

BoISLÈVE J., collab. RothÉ M.-P., GENOT A., 2014b, « Nouvelles découvertes sur le site de la Verrerie à Arles : de remarquables peintures murales de deuxième style pompéien », in CAPUS P., DARDENAY A. (éd.), L'empire de la couleur, de Pompéi au sud des Gaules, Toulouse, p. I-V.

BoISLÈVE J., 2014C, « Derrière l'enduit, l'architecture », in DARDENAY A. (coord.), L'empire de la couleur : La peinture romaine de Pompéi aux Gaules, Dossiers d'Archéologie, n 366, p. 76-77.

BOISLÈVE J., 2014d, « L'étude toichographologique », in Ronco C. (dir.), Die, Drôme, Rhône-Alpes, place de la Cathédrale - place du Marché/ aménagement des places, Rapport d'opération, Inrap-SRA Rhône-Alpes, vol. 1, 147-188, vol. 2, 79-192, vol. 3, 145-205.

BOISLÈVE J., 2016, « Terre et peinture à l'époque romaine. Pratiques architecturales et décoratives en Gaule romaine mises en évidence par l'analyse toichographologique ", Archéopages, 42, p. 48-57.

Boislève J., Allag C., 2011, « Un décor stuqué monumental du Bas-Empire à Autun », Gallia, 68-2, p. 195-235.

Boislève J., Breuil J.-Y., Coutelas A., Vauxion O., 2008, « La prise en compte des enduits peints lors des opérations d'archéologie préventive : la relève d'un important corpus d'enduits à Nîmes ", Archéopages, Limites et territoire, $\mathrm{n}^{\circ}$ 21, p. 64-67.

Boislève J., Breuil J.-Y., Houix B., Vauxion O., 2011, "Mosaïques et peintures d'un quartier de Nemausus, La fouille du parking Jean-Jaurès à Nîmes (Gard) », in BALMelle et al., 2011, p. 33-48.

Boislève J., Cayn P., collab. Tendron G., Fuchs M., 2013, « Les fouilles du quartier Clérisseau à Nîmes : analyse des décors d'une vaste domus », Revue archéologique de Narbonnaise, 45, p. 257-291.

Boislève J., Chuniaud K., 2014, « Les peintures du sanctuaire de Trémonteix à Clermont-Ferrand (Puy-de-Dôme) », in BorsLÈve et al., 2014, p. 157-177.
Boislève J., Dardenay A., Monier F. (éd.), 2016 Peintures et stucs d'époque romaine : une archéologie du décor, Actes du $27^{e}$ colloque de l'AFPMA, Toulouse, 21 et 22 novembre 2014, Bordeaux, Ausonius (Pictor collection de l'AFPMA, 5), $427 \mathrm{p}$.

Boislève J., Dardenay A., Monier F. (éd.), 2014, Peintures et stucs d'époque romaine : révéler l'architecture par l'étude du décor, Actes du 26 colloque de l'AFPMA, Strasbourg, 16 et 17 novembre 2012, Bordeaux, Ausonius (Pictor collection de l'AFPMA, 3), 345 p.

Boislève J., Dardenay A., Monier F. (éd.), 2013 Peintures murales et stucs d'époque romaine, De la fouille au musée, Actes des $24^{e}$ et $25^{e}$ colloques de l'AFPMA, Narbonne 12 et 13 novembre 2010 et Paris, 25 et 26 novembre 2011, Bordeaux, Ausonius (Pictor collection de l'AFPMA, 1), 493 p.

Boislève J., Groetembril S., Vibert-Guigue C., 2007, "Les peintures de la villa de Mané-Véchen (France) », in Guiral, Pelegrin, 2007, p. 433-435, pl. 40.

Boislève J., RonCo C., 2016, «Pan, Bacchus et le sphinx. Une peinture murale d'époque romaine découverte à Die (Drôme) », in BoISLÈve et al., 2016, p. 37-58.

Guiral Pelegrin C. (éd.), 2007, Circulation de temas y sistemas decorativos en la pintura mural antigua, actes du colloque international de l'AIPMA, 21-25 septembre 2004, Saragosse, Saragosse, 559 p.

Guiral Pelegrin C., 2010, « La decoración pintada del "cubiculo de las estaciones" de la villa romana dels Munts (Altafulla, Tarragona) », Espacio, Tiempo y Forma, serie I. nueva epoca. Prehistoria y Arqueologia, t. 3, p. 127-144. 